\title{
ON G-RADICAL SUPPLEMENT SUBMODULES
}

\author{
HASAN HÜSEYIN ÖKTEN AND AYTEN PEKIN
}

Received 23 June, 2020

\begin{abstract}
In this work, we give some new properties of Rad-supplement and g-radical supplement submodules. Let $V$ be a g-radical supplement of $U$ in $M$ and $U$ or $V$ be essential submodule of $M$. Then $\operatorname{Rad}_{g} V=V \cap \operatorname{Rad}_{g} M$. Let $V$ be a g-radical supplement of $U$ in $M, U$ or $V$ be essential submodule of $M$ and $x \in V$. Then $R x \ll_{g} V$ if and only if $R x \ll_{g} M$. In this work, some relations between Rad-supplement, g-radical supplement, $\beta^{*}$ and $\beta_{g}^{*}$ relations are also studied. Let $X \beta_{g}^{*} Y$ in $M$. If $V$ is a g-radical supplement of $X$ in $M$ and $V \unlhd M$, then $V$ is also a g-radical supplement of $Y$ in $M$. Let $M$ be an $R$-module. It is proved that $M$ is semilocal (g-semilocal) if every submodule of $M \beta^{*}$ equivalent to a Rad-supplement (g-radical supplement) submodule in $M$.
\end{abstract}

2010 Mathematics Subject Classification: 16D10; 16D70

Keywords: small submodules, radical, g-supplemented modules, g-radical supplemented modules

\section{INTRODUCTION}

Throughout this paper all rings will be associative with identity and all modules will be unital left modules.

Let $R$ be a ring and $M$ be an $R$-module. We will denote a submodule $N$ of $M$ by $N \leq M$. Let $M$ be an $R$-module and $N \leq M$. If $L=M$ for every submodule $L$ of $M$ such that $M=N+L$, then $N$ is called a small submodule of $M$ and denoted by $N \ll M$. Let $M$ be an $R$-module and $N \leq M$. If there exists a submodule $K$ of $M$ such that $M=N+K$ and $N \cap K=0$, then $N$ is called a direct summand of $M$ and it is denoted by $M=N \oplus K$. A submodule $N$ of an $R$-module $M$ is called an essential submodule of $M$, denoted by $N \unlhd M$, in case $K \cap N \neq 0$ for every submodule $K \neq 0$, or equivalently, $N \cap K=0$ implies that $K=0$. Let $M$ be an $R$-module and $K$ be a submodule of $M . K$ is called a generalized small (briefly, $g$-small) submodule of $M$ if for every $T \unlhd M$ with $M=K+T$ implies that $T=M$, this is written by $K \ll_{g} M$ (in [13], it is called an $e$-small submodule of $M$ and denoted by $K \ll_{e} M$ ). It is clear that every small submodule is a generalized small submodule but the converse is not true generally. Let $U$ and $V$ be submodules of $M$. If $M=U+V$ and $V$ is minimal with respect to this property, or equivalently, $M=U+V$ and $U \cap V<<V$, then $V$ is called a supplement of $U$ in $M . M$ is called a supplemented module if every 
submodule of $M$ has a supplement in $M$. Let $M$ be an $R$-module and $U, V \leq M$. If $M=U+V$ and $M=U+T$ with $T \unlhd V$ implies that $T=V$, or equivalently, $M=U+V$ and $U \cap V \ll_{g} V$, then $V$ is called a $g$-supplement of $U$ in $M . M$ is said to be $g$-supplemented if every submodule of $M$ has a g-supplement in $M$. The intersection of all maximal submodules of an $R$-module $M$ is called the radical of $M$ and denoted by $\operatorname{Rad} M$. If $M$ have no maximal submodules, then we denote $\operatorname{Rad} M=M . M$ is said to be semilocal if $M / \operatorname{Rad} M$ is semisimple, i. e. every submodule of $M / \operatorname{Rad} M$ is a direct summand of $M / \operatorname{Rad} M$. Let $M$ be an $R$-module and $U, V \leq M$. If $M=U+V$ and $U \cap V \leq R a d V$, then $V$ is called a generalized (radical) supplement (briefly Rad-supplement) of $U$ in $M . M$ is said to be generalized (radical) supplemented (briefly Rad-supplemented) if every submodule of $M$ has a Rad-supplement in $M$. The intersection of all essential maximal submodules of an $R$-module $M$ is called the generalized radical (briefly g-radical) of $M$ and denoted by $\operatorname{Rad}_{g} M$ (in [13], it is denoted by $\operatorname{Rad}_{e} M$ ). If $M$ have no essential maximal submodules, then we denote $\operatorname{Rad}_{g} M=M$. If $M / \operatorname{Rad}_{g} M$ is semisimple, i. e. every submodule of $M / \operatorname{Rad}_{g} M$ is a direct summand of $M / \operatorname{Rad}_{g} M$, then $M$ is called a $g$-semilocal module. Let $M$ be an $R$-module. We say submodules $X$ and $Y$ of $M$ are $\beta^{*}$ equivalent, $X \beta^{*} Y$, if and only if $Y+K=M$ for every $K \leq M$ such that $X+K=M$ and $X+T=M$ for every $T \leq M$ such that $Y+T=M$. We say submodules $X$ and $Y$ of $M$ are $\beta_{g}^{*}$ equivalent, $X \beta_{g}^{*} Y$, if and only if $Y+K=M$ for every $K \unlhd M$ such that $X+K=M$ and $X+T=M$ for every $T \unlhd M$ such that $Y+T=M$. Let $M$ be an $R$-module and $X \leq Y \leq M$. If $Y / X \ll M / X$, then we say $Y$ lies above $X$ in $M$.

More informations about supplemented modules are in $[2,8,12]$. More informations about g-small submodules and g-supplemented modules are in $[3,7,10]$. The definition of generalized supplemented modules and some properties of them are in [11]. The definition of g-semilocal modules and some properties of them are in [5]. The definition of $\beta^{*}$ relation and some results of this relation are in [1]. The definition of $\beta_{g}^{*}$ relation and some results of this relation are in [9].

Lemma 1. Let $M$ be an R-module. The following assertions hold.

(1) For every $m \in \operatorname{Rad}_{g} M, R m \ll_{g} M$.

(2) If $N \leq M$, then $\operatorname{Rad}_{g} N \leq \operatorname{Rad}_{g} M$.

(3) $\operatorname{Rad}_{g} M=\sum_{L \ll_{g} M} L$.

Proof. See [4, Lemma 2 and Lemma 3].

\section{G-RADICAL SUPPLEMENT SUBMODULES}

Definition 1. Let $M$ be an $R$-module and $U, V \leq M$. If $M=U+V$ and $U \cap V \leq$ $\operatorname{Rad}_{g} V$, then $V$ is called a g-radical supplement of $U$ in $M$. If every submodule of $\bar{M}$ has a g-radical supplement in $M$, then $M$ is called a g-radical supplemented module. $($ See $[4,6]$.) 
Clearly we can see that every g-supplemented module is g-radical supplemented. But the converse is not true in general. Every Rad-supplemented module is g-radical supplemented.

Lemma 2. Let $V$ be a g-radical supplement of $U$ in $M$ and $U \unlhd M$. Then $\operatorname{Rad}_{g} V=V \cap \operatorname{Rad}_{g} M$.

Proof. By Lemma 1, $\operatorname{Rad}_{g} V \leq V \cap \operatorname{Rad}_{g} M$. Let $T$ be an essential maximal submodule of $V$. Then $U \cap V \leq \operatorname{Rad}_{g} V \leq T$ holds. By $\frac{M}{U+T}=\frac{U+T+V}{U+T} \cong \frac{V}{V \cap(U+T)}=$ $=\frac{V}{U \cap V+T}=\frac{V}{T}$ and $U+T \unlhd M, U+T$ is an essential maximal submodule of $M$ and $\operatorname{Rad}_{g} M \leq U+T$. Hence $V \cap \operatorname{Rad}_{g} M \leq V \cap(U+T)=U \cap V+T=T$. Thus $\operatorname{Rad}_{g} V=V \cap \operatorname{Rad}_{g} M$, as desired.

Theorem 1. Let $V$ be a g-radical supplement of $U$ in $M, U \unlhd M$ and $x \in V$. Then $R x \ll_{g} V$ if and only if $R x \ll_{g} M$.

Proof.

$\Longrightarrow$ Clear.

$\Longleftarrow$ Since $R x \ll_{g} M$, by Lemma $1, R x \leq \operatorname{Rad}_{g} M$ and $x \in \operatorname{Rad}_{g} M$. Then $x \in V \cap \operatorname{Rad}_{g} M$. By Lemma $2, \operatorname{Rad}_{g} V=V \cap \operatorname{Rad}_{g} M$. Hence $x \in \operatorname{Rad}_{g} V$ and by Lemma $1, R x \ll_{g} V$. We can also prove this part as follows:

Let $T$ be an essential maximal submodule of $V$. Here $U \cap V \leq \operatorname{Rad}_{g} V \leq T$. Assume that $R x \leq T$. Then $R x+T=V$ and $M=U+V=U+R x+T$. Since $R x \ll_{g} M$ and $U+T \unlhd M, U+T=M$. Then $V=V \cap M=V \cap(U+T)=$ $=U \cap V+T=T$, a contradiction. Hence $R x \leq T$ for every essential maximal submodule $T$ of $V$ and $R x \leq \operatorname{Rad}_{g} V$. Thus $x \in \operatorname{Rad}_{g} V$ and by Lemma 1 , $R x \ll_{g} V$.

Corollary 1. Let $V$ be a Rad-supplement of $U$ in $M$ and $U \unlhd M$. Then $\operatorname{Rad}_{g} V=V \cap \operatorname{Rad}_{g} M$.

Proof. Clear from Lemma 2.

Corollary 2. Let $V$ be a Rad-supplement of $U$ in $M, U \unlhd M$ and $x \in V$. Then $R x \ll_{g} V$ if and only if $R x \ll_{g} M$.

Proof. Clear from Theorem 1.

Theorem 2. Let $V$ be a g-radical supplement of $U$ in $M, V \unlhd M$ and $x \in V$. The following assertions hold.

(1) $\operatorname{Rad}_{g} V=V \cap \operatorname{Rad}_{g} M$.

(2) $R x \ll_{g} V$ if and only if $R x \ll_{g} M$.

Proof. 
(1) By Lemma $1, \operatorname{Rad}_{g} V \leq V \cap \operatorname{Rad}_{g} M$. Let $T$ be an essential maximal submodule of $V$. Then $U \cap V \leq \operatorname{Rad}_{g} V \leq T$ holds. Since $T \unlhd V$ and $V \unlhd M$, then $T \unlhd M$ and $U+T \unlhd M$. Then by $\frac{M}{U+T}=\frac{U+T+V}{U+T} \cong \frac{V}{V \cap(U+T)}=\frac{\bar{V}}{U \cap V+T}=\frac{V}{T}$, $U+T$ is an essential maximal submodule of $M$ and $\operatorname{Rad}_{g} M \leq U+T$. Hence $V \cap \operatorname{Rad}_{g} M \leq V \cap(U+T)=U \cap V+T=T$. Thus $\operatorname{Rad}_{g} V=V \cap \operatorname{Rad}_{g} M$, as desired.

(2) $\Longrightarrow$ Clear.

$\Longleftarrow$ Since $R x \ll_{g} M$, by Lemma $1, R x \leq \operatorname{Rad}_{g} M$ and $x \in \operatorname{Rad}_{g} M$. Then $x \in V \cap \operatorname{Rad}_{g} M$. By Theorem 2 (1), $\operatorname{Rad}_{g} V=V \cap \operatorname{Rad}_{g} M$. Hence $x \in \operatorname{Rad}_{g} V$ and by Lemma $1, R x \ll_{g} V$. We can also prove this part as follows:

Let $T$ be an essential maximal submodule of $V$. Here $U \cap V \leq \operatorname{Rad}_{g} V \leq$ $\leq T$. Assume that $R x \not T$. Then $R x+T=V$ and $M=U+V=$ $=U+R x+T$. Since $T \unlhd V$ and $V \unlhd M$, then $T \unlhd M$ and $U+T \unlhd M$. Since $R x \ll_{g} M, U+T=M$. Then $V=V \cap M=V \cap(U+T)=$ $=U \cap V+T=T$, a contradiction. Hence $R x \leq T$ for every essential maximal submodule $T$ of $V$ and $R x \leq \operatorname{Rad}_{g} V$. Thus $x \in \operatorname{Rad}_{g} V$ and by Lemma $1, R x \ll_{g} V$.

Corollary 3. Let $V$ be a Rad-supplement of $U$ in $M$ and $V \unlhd M$. Then $\operatorname{Rad}_{g} V=V \cap \operatorname{Rad}_{g} M$.

Proof. Clear from Theorem 2 (1).

Corollary 4. Let $V$ be a Rad-supplement of $U$ in $M, V \unlhd M$ and $x \in V$. Then $R x \ll_{g} V$ if and only if $R x \ll_{g} M$.

Proof. Clear from Theorem 2 (2).

Example 1. Consider the $\mathbb{Z}$-module $\mathbb{Q}$. For $\mathbb{Z} \leq_{\mathbb{Z}} \mathbb{Q}, \operatorname{Rad}_{g} \mathbb{Z}=\operatorname{Rad} \mathbb{Z}=0$. Since $\operatorname{Rad}_{g} \mathbb{Q}=\operatorname{Rad} \mathbb{Q}=\mathbb{Q}, \mathbb{Z} \cap \operatorname{Rad}_{g} \mathbb{Q}=\mathbb{Z} \cap \mathbb{Q}=\mathbb{Z}$. Hence $\operatorname{Rad}_{g} \mathbb{Z} \neq \mathbb{Z} \cap \operatorname{Rad}_{g} \mathbb{Q}$.

Proposition 1. Let $X \beta_{g}^{*} Y$ in $M$. If $V$ is a g-radical supplement of $X$ in $M$ and $V \unlhd M$, then $V$ is also a g-radical supplement of $Y$ in $M$.

Proof. By hypothesis, $M=X+V$ and $X \cap V \leq \operatorname{Rad}_{g} V$. Since $X \beta_{g}^{*} Y$ and $V \unlhd M$, $Y+V=M$. Let $T$ be any essential maximal submodule of $V$. Since $T \unlhd V$ and $V \unlhd M$, then $T \unlhd M$. Assume that $Y \cap V \not \leq T$. Then $Y \cap V+T=V$. Here $M=$ $=Y+V=Y+Y \cap V+T=Y+T$ and since $X \beta_{g}^{*} Y$ and $T \unlhd M, X+T=M$. Then $V=$ $V \cap M=V \cap(X+T)=V \cap X+T$ and since $X \cap V \leq \operatorname{Rad}_{g} V \leq T, V=V \cap X+T=T$. This is a contradiction. Hence $Y \cap V \leq T$ for every essential maximal submodule of $V$ and $Y \cap V \leq \operatorname{Rad}_{g} V$. Thus $V$ is a g-radical supplement of $Y$ in $M$.

Lemma 3. Let $X \beta^{*} Y$ in $M$. If $X$ and $Y$ have Rad-supplements in $M$, then they have the same Rad-supplements in $M$. 
Proof. Let $C$ be a Rad-supplement of $X$ in $M$. Then $M=X+C$ and $X \cap C \leq R a d C$. Since $X \beta^{*} Y, Y+C=M$. Let $T$ be any maximal submodule of $C$. Assume that $Y \cap C \not \leq T$. Then $Y \cap C+T=C$. Here $M=Y+C=Y+Y \cap C+T=Y+T$ and since $X \beta^{*} Y, X+T=M$. Then $C=C \cap M=C \cap(X+T)=X \cap C+T$ and since $X \cap C \leq \operatorname{Rad} C \leq T, C=X \cap C+T=T$. This is a contradiction. Hence $Y \cap C \leq T$ for every maximal submodule of $C$ and $Y \cap C \leq \operatorname{Rad} C$. Thus $C$ is a Rad-supplement of $Y$ in $M$. Similarly, the interchanging the roles of $X$ and $Y$, we can prove that every Rad-supplement of $Y$ in $M$ is also a Rad-supplement of $X$ in $M$.

Corollary 5. Let $X$ lies above $Y$ in $M$. If $X$ and $Y$ have Rad-supplements in $M$, then they have the same Rad-supplements in $M$.

Proof. Clear from Lemma 3.

Lemma 4. Let $X \beta^{*} Y$ in $M$. If $X$ has a g-radical supplement $V$ in $M$, then $V$ is also a g-radical supplement of $Y$ in $M$.

Proof. By hypothesis, $M=X+V$ and $X \cap V \leq \operatorname{Rad}_{g} V$. Since $X \beta^{*} Y, Y+V=M$. Let $T$ be any essential maximal submodule of $V$. Assume that $Y \cap V \not \leq T$. Then $Y \cap V+T=V$. Here $M=Y+V=Y+Y \cap V+T=Y+T$ and since $X \beta^{*} Y$, $X+T=M$. Then $V=V \cap M=V \cap(X+T)=X \cap V+T$ and since $X \cap V \leq$ $\leq \operatorname{Rad}_{g} V \leq T, V=V \cap X+T=T$. This is a contradiction. Hence $Y \cap V \leq T$ for every essential maximal submodule of $V$ and $Y \cap V \leq \operatorname{Rad}_{g} V$. Thus $V$ is a g-radical supplement of $Y$ in $M$.

Corollary 6. Let $X$ lies above $Y$ in $M$. If $X$ and $Y$ have g-radical supplements in $M$, then they have the same $g$-radical supplements in $M$.

Proof. Clear from Lemma 4.

Lemma 5. Let $X \beta^{*} Y$ and $Y$ be a Rad-supplement of $U$ in $M$. Then $U \cap X \leq R a d M$.

Proof. Since $Y$ is a Rad-supplement of $U$ in $M, M=U+Y$ and $U \cap Y \leq \operatorname{Rad} Y \leq$ $\leq \operatorname{Rad} M$. Since $M=U+Y$ and $X \beta^{*} Y, M=U+X$. Let $T$ be any maximal submodule of $M$. Here $U \cap Y \leq R a d M \leq T$. Assume that $U \cap X \not \leq T$. Then $U \cap X+T=M$ and since $M=U+X$, by [2, Lemma 1.24], $X+U \cap T=M$. Since $X \beta^{*} Y, Y+U \cap T=M$ and since $U+T=M$, by [2, Lemma 1.24] again, $U \cap Y+T=M$. Then by $U \cap Y \leq T$, $M=U \cap Y+T=T$. This is a contradiction. Hence $U \cap X \leq T$ for every maximal submodule $T$ of $M$ and $U \cap X \leq R a d M$.

Corollary 7. Let $X$ lies above $Y$ and $Y$ be a Rad-supplement of $U$ in $M$. Then $U \cap X \leq R a d M$.

Proof. Clear from Lemma 5.

Lemma 6. Let $M$ be an R-module. If every submodule of $M$ is $\beta^{*}$ equivalent to a Rad-supplement submodule in $M$, then $M$ is semilocal. 
Proof. Let $X / \operatorname{Rad} M \leq M / \operatorname{Rad} M$. Since $X \leq M$, by hypothesis, there exists a Rad-supplement submodule $Y$ in $M$ such that $X \beta^{*} Y$. Let $Y$ be a Rad-supplement of $U$ in $M$. By Lemma 5, $U \cap X \leq \operatorname{Rad} M$. Since $X \beta^{*} Y$ and $Y+U=M, X+U=M$. Then $\frac{M}{\operatorname{Rad} M}=\frac{X+U}{\operatorname{Rad} M}=\frac{X}{\operatorname{Rad} M}+\frac{U+\operatorname{Rad} M}{\operatorname{Rad} M}$ and $\frac{X}{\operatorname{Rad} M} \cap \frac{U+\operatorname{Rad} M}{\operatorname{Rad} M}=\frac{X \cap(U+\operatorname{Rad} M)}{\operatorname{Rad} M}=\frac{U \cap X+\operatorname{Rad} M}{\operatorname{Rad} M}=$ $=\frac{\operatorname{Rad} M}{\operatorname{Rad} M}=0$. Hence $\frac{M}{\operatorname{Rad} M}=\frac{X}{\operatorname{Rad} M} \oplus \frac{U+\operatorname{Rad} M}{\operatorname{Rad} M}$ and $M / \operatorname{Rad} M$ is semisimple. Thus $M$ is semilocal.

Corollary 8. Let $M$ be an R-module. If every submodule of $M$ lies above a Rad-supplement submodule in $M$, then $M$ is semilocal.

Proof. Clear from Lemma 6.

Theorem 3. Let $X \beta^{*} Y$ and $Y$ be a g-radical supplement of $U$ in $M$. Then $U \cap X \leq \operatorname{Rad}_{g} M$.

Proof. Since $Y$ is a g-radical supplement of $U$ in $M, M=U+Y$ and $U \cap Y \leq$ $\leq \operatorname{Rad}_{g} Y \leq \operatorname{Rad}_{g} M$. Since $M=U+Y$ and $X \beta^{*} Y, M=U+X$. Let $T$ be any essential maximal submodule of $M$. Here $U \cap Y \leq \operatorname{Rad}_{g} M \leq T$. Assume that $U \cap X \not \leq T$. Then $U \cap X+T=M$ and since $M=U+X$, by [2, Lemma 1.24], $X+U \cap T=M$. Since $X \beta^{*} Y, Y+U \cap T=M$ and since $U+T=M$, by [2, Lemma 1.24] again, $U \cap Y+T=M$. Then by $U \cap Y \leq T, M=U \cap Y+T=T$. This is a contradiction. Hence $U \cap X \leq T$ for every essential maximal submodule $T$ of $M$ and $U \cap X \leq \operatorname{Rad}_{g} M$.

Corollary 9. Let $X$ lies above $Y$ and $Y$ be a g-radical supplement of $U$ in $M$. Then $U \cap X \leq \operatorname{Rad}_{g} M$.

Proof. Clear from Theorem 3.

Theorem 4. Let $M$ be an R-module. If every submodule of $M$ is $\beta^{*}$ equivalent to a g-radical supplement submodule in $M$, then $M$ is $g$-semilocal.

Proof. Let $X / \operatorname{Rad}_{g} M \leq M / \operatorname{Rad}_{g} M$. Since $X \leq M$, by hypothesis, there exists a g-radical supplement submodule $Y$ in $M$ such that $X \beta^{*} Y$. Let $Y$ be a g-radical supplement of $U$ in $M$. By Theorem 3, $U \cap X \leq \operatorname{Rad}_{g} M$. Since $X \beta^{*} Y$ and $Y+U=M$, $X+U=M$. Then $\frac{M}{\operatorname{Rad}_{g} M}=\frac{X+U}{\operatorname{Rad}_{g} M}=\frac{X}{\operatorname{Rad}_{g} M}+\frac{U+\operatorname{Rad}_{g} M}{\operatorname{Rad}_{g} M}$ and $\frac{X}{\operatorname{Rad}_{g} M} \cap \frac{U+\operatorname{Rad}_{g} M}{\operatorname{Rad}_{g} M}=$ $=\frac{X \cap\left(U+\operatorname{Rad}_{g} M\right)}{\operatorname{Rad}_{g} M}=\frac{U \cap X+\operatorname{Rad}_{g} M}{\operatorname{Rad}_{g} M}=\frac{\operatorname{Rad}_{g} M}{\operatorname{Rad}_{g} M}=0$. Hence $\frac{M}{\operatorname{Rad}_{g} M}=\frac{X}{\operatorname{Rad}_{g} M} \oplus \frac{U+\operatorname{Rad}_{g} M}{\operatorname{Rad}_{g} M}$ and $M / \operatorname{Rad}_{g} M$ is semisimple. Thus $M$ is g-semilocal.

Corollary 10. Let $M$ be an R-module. If every submodule of $M$ lies above a $g$ radical supplement submodule in $M$, then $M$ is g-semilocal.

Proof. Clear from Theorem 4. 


\section{REFERENCES}

[1] G. F. Birkenmeier, F. T. Mutlu, C. Nebiyev, N. Sokmez, and A. Tercan, "Goldie*supplemented modules," Glasgow Mathematical Journal, vol. 52A, pp. 41-52, 2010, doi: 10.1017/s0017089510000212.

[2] J. Clark, C. Lomp, N. Vanaja, and R. Wisbauer, Lifting Modules: Supplements and Projectivity in Module Theory (Frontiers in Mathematics), 2006th ed. Basel: Birkhäuser, $82006 . \quad$ doi: 10.1007/3-7643-7573-6.

[3] B. Koşar, C. Nebiyev, and N. Sökmez, "G-supplemented modules," Ukrainian Mathematical Journal, vol. 67, no. 6, pp. 861-864, 2015, doi: 10.1007/s11253-015-1127-8.

[4] B. Koşar, C. Nebiyev, and A. Pekin, "A generalization of g-supplemented modules," Miskolc Mathematical Notes, vol. 20, no. 1, pp. 345-352, 2019, doi: 10.18514/mmn.2019.2586.

[5] C. Nebiyev and H. H. Ökten, "Weakly g-supplemented modules," European Journal of Pure and Applied Mathematics, vol. 10, no. 3, pp. 521-528, 2017.

[6] C. Nebiyev, "g-radical supplemented modules," in Antalya Algebra Days XVII, Şirince, İzmir, Turkey, 2015.

[7] C. Nebiyev, "On a generalization of supplement submodules," International Journal of Pure and Applied Mathematics, vol. 113, no. 2, pp. 283-289, 2017, doi: 10.12732/ijpam.v113i2.8.

[8] C. Nebiyev and A. Pancar, "On supplement submodules," Ukrainian Mathematical Journal, vol. 65 , no. 7, pp. 1071-1078, 2013, doi: 10.1007/s11253-013-0842-2.

[9] C. Nebiyev and N. Sökmez, "Beta g-star relation on modules," European Journal of Pure and Applied Mathematics, vol. 11, no. 1, pp. 238-243, 2018.

[10] N. Sökmez, B. Koşar, and C. Nebiyev, "Genelleştirilmiş küçük alt modüller," in XIII. Ulusal Matematik Sempozyumu. Kayseri: Erciyes Üniversitesi, 2010.

[11] Y. Wang and N. Ding, "Generalized supplemented modules," Taiwanese Journal of Mathematics, vol. 10, no. 6, pp. 1589-1601, 2006, doi: 10.11650/twjm/1500404577.

[12] R. Wisbauer, Foundations of Module and Ring Theory. Philadelphia: Gordon and Breach, 1991. doi: 10.1201/9780203755532.

[13] D. X. Zhou and X. R. Zhang, "Small-essential submodules and morita duality," Southeast Asian Bulletin of Mathematics, vol. 35, pp. 1051-1062, 2011.

Authors' addresses

\section{Hasan Hüseyin Ökten}

Amasya University, Technical Sciences Vocational School, Amasya, Turkey

E-mail address: hokten@gmail.com

Ayten Pekin

Turkey

(Corresponding author) Istanbul University, Department of Mathematics, Vezneciler, Istanbul,

E-mail address: aypekin@istanbul.edu.tr, aspekin@hotmail.com 